\title{
Control of Hand Impedance under Static Conditions and During Reaching Movement
}

\author{
Mohammad Darainy ${ }^{1,2}$, Farzad Towhidkhah ${ }^{3}$ and \\ David J. Ostry ${ }^{1,4}$ \\ ${ }^{1}$ McGill University, Montreal, Quebec \\ ${ }^{2}$ Shahed University, Tehran, Iran \\ ${ }^{3}$ AmirKabir University of Technology, Tehran, Iran \\ ${ }^{4}$ Haskins Laboratories, New Haven, Connecticut
}

\author{
Mailing Address: $\quad$ D. J. Ostry \\ Department of Psychology \\ McGill University \\ 1205 Dr. Penfield Avenue \\ Montreal, QC, Canada H3A 1B1 \\ Phone: 5143986111 \\ Fax: 5143984896 \\ E-mail: ostry@motion.psych.mcgill.ca
}

Running head: Hand impedance in statics and during movement 


\begin{abstract}
It is known that humans can modify the impedance of the musculoskeletal periphery but the extent of this modification is uncertain. Previous studies on impedance control under static conditions indicate a limited ability to modify impedance, whereas studies of impedance control during reaching in unstable environments suggest a greater range of impedance modification. As a first step in accounting for this difference, we have quantified the extent to which stiffness changes from posture to movement even when there are no destabilizing forces. Hand stiffness was estimated under static conditions and at the same position during both longitudinal (near to far) and lateral movements using a position-servo technique. A new method was developed to predict the hand "reference" trajectory for purposes of estimating stiffness. For movements in a longitudinal direction, there was considerable counter-clockwise rotation of the hand stiffness ellipse relative to stiffness under static conditions. In contrast, a small counterclockwise rotation was observed during lateral movement. In the modeling studies, even when we used the same modeled cocontraction level during posture and movement, we found that there was a substantial difference in the orientation of the stiffness ellipse, comparable to that observed empirically. Indeed, the main determinant of the orientation of the ellipse in our modeling studies was the movement direction and the muscle activation associated with movement. Changes in the cocontraction level and the balance of cocontraction had smaller effects. Thus even when there is no environmental instability, the orientation of stiffness ellipse changes during movement in a manner that varies with movement direction.
\end{abstract}




\section{INTRODUCTION}

Impedance control, the idea that the nervous system can modify the mechanical behavior of neuromuscular periphery to control limb movement and posture (Hogan 1985), is a focus of interest as a potential neural means to aid in the achievement of stability. There have been a number of reports indicating that subjects can voluntarily modify stiffness patterns to provide stability in opposition to load. However, the apparent capacity for impedance adjustments seems far greater during movement than when subjects alter impedance under static conditions. Here we have compared hand stiffness during movement with stiffness measured at the same location when the limb is stationary. The goal is to assess the extent to which impedance changes simply as a result of limb movement.

Using EMG biofeedback, Gomi and Osu (1998) showed that subjects can change the orientation of the stiffness ellipse. When the manipulandum was held with the right hand, elbow cocontraction produced clockwise rotation; shoulder cocontraction produced counter-clockwise rotation. Perreault et al. (2002) report a similar pattern of stiffness change in response to a real-time display of a stiffness ellipse. Darainy et al. (2004) investigated the ability to modify arm stiffness under static conditions using kinaesthetic information. Clockwise rotation of the stiffness ellipse was observed in response to force pulses along a longitudinal axis. Counter-clockwise rotation was observed for force pulses along a lateral axis. In each of these studies, the maximum change in ellipse orientation was 30 deg or less. Changes in stiffness orientation during movement are 
larger. Burdet et al. (2001) and Franklin et al. (2003) estimated stiffness during adaptation to divergent force-fields. They show that changes in impedance closely match the direction of load such that the orientation of maximum stiffness at the hand approaches the direction of the environmental instability.

Impedance measurement is a challenging problem during movement. Gomi and Kawato (1997) described a technique for stiffness estimation during reaching movements. The hand was perturbed using force pulses and resulting displacements were used to estimate stiffness. Position servo-control is another approach to stiffness estimation. Its advantage is that a direct estimate of stiffness can be obtained without the necessity of estimating viscosity simultaneously. However the success of the technique is dependent upon a precise algorithm that predicts where the arm would be if the positionservo was inactive.

In this study we have developed a new method of position servo-control for stiffness estimation during reaching movements. We use the mean of previous movements along with an auto-regressive (AR) model to predict the difference between the mean and upcoming movement trajectory. Displacements are position servocontrolled relative to this trajectory that is predicted in real-time. We estimated stiffness at the same location in the center of workspace during postural maintenance, and during lateral and longitudinal movements. We observed that even in the absence of external load, there is a considerable counter-clockwise rotation of the stiffness ellipse during longitudinal movements relative to value measured when the limb is stationary. The 
empirical procedure was simulated using a model of two-joint arm movement. In the modeling studies, we found that even when the modeled cocontraction level was the same during posture and movement, there was a rotation of the hand stiffness ellipse that was comparable to that observed empirically. This suggests that even in the absence of destabilizing loads there is a change in ellipse orientation during movement that varies with movement direction and is thus presumably related to the forces that transport the limb.

\section{MATERIALS AND METHODS}

Subjects and apparatus. Six right-handed subjects, between 19 and 31, participated in this study. The subjects were seated in front of two degree-of-freedom robotic arm and held the handle with their right hand (Figure 1). Joint angles were measured with optical encoders in the robot arm (Gurley Precision Instruments). Force signals were measured with a force-torque sensor (ATI Industrial Automation) that was mounted just above the handle of the manipulandum. Visual feedback of subject's arm was provided during all phases of experiment. The subject's arm was supported against the gravity by an air-sled. Shoulder movement was restricted by a harness and the wrist was braced. A computer monitor was placed in front of subject. During the estimation of stiffness under static conditions a $15 \mathrm{~mm}$ red circle in the middle of monitor represented the target location and a $10 \mathrm{~mm}$ yellow circle specified hand position. During reaching movements, $15 \mathrm{~mm}$ white circles were used to represent the movement start and end points; subjects' hand position was represented by a $10 \mathrm{~mm}$ green circle. 
Estimation of stiffness under static conditions. Subjects were placed in a standard position such that the elbow angle was $90 \mathrm{deg}$ and the shoulder angle was $45 \mathrm{deg}$. Subjects were required to place the hand in the middle of the target zone and to remain on target. Subjects were instructed not to intervene in any manner.

We used position servo-control to estimate stiffness (Darainy et al., 2004). The amplitude of the displacement was $10 \mathrm{~mm}$ (mean actual displacement was $9.9 \pm 0.1 \mathrm{~mm}$ ) and hold phase of the displacement was $200 \mathrm{~ms}$. The displacement was ramped on (and off) over $100 \mathrm{~ms}$ to give a total duration of $400 \mathrm{~ms}$ for the displacement. The subject's hand was randomly displaced in each of eight directions and restoring forces and displacements were recorded. During servo-displacement, stiffness and damping of the manipulandum were $6000 \mathrm{~N} / \mathrm{m}$ and $450 \mathrm{~N} . \mathrm{s} / \mathrm{m}$, respectively. Visual feedback of hand was frozen during this displacement. Prior to each displacement, the subject's hand had to be within the target zone and hand velocity had to be less than $1 \mathrm{~mm} / \mathrm{sec}$. Once these conditions were satisfied, a measurement was initiated at a randomly selected time between 0.5 and 1.5 seconds. Position and force data from the $50 \mathrm{~ms}$ preceding the measurement displacement and from the final $50 \mathrm{~ms}$ of the hold phase $(250 \mathrm{~ms}$ from the start of the position servo interval) were used to estimate stiffness. Thirty two trials (four trials in each of eight directions) were recorded for stiffness estimation under static conditions. 
Estimation of stiffness during movement. Subjects were tested in two experimental conditions (near to far and left to right movements) over three sessions, one for familiarization and one each for the two experimental conditions. In each session subjects were instructed to make $35 \mathrm{~cm}$ movements to a $15 \mathrm{~mm}$ target. Subjects were trained to produce movements of $1200 \pm 50 \mathrm{msec}$ and were asked to move as straight as possible. Visual feedback on movement duration was provided. The actual average movement duration was $1210 \pm 90 \mathrm{msec}$. Each experimental block involved eighty trials in a single direction. In the familiarization session four blocks of trials were run without any perturbations (two blocks in each direction). One further block of practice trials included servo-displacements like those used for estimation of stiffness under static conditions. The displacements were interspersed randomly in $20 \%$ of trials and subjects were instructed not to intervene.

In each of two sessions, arm stiffness was estimated for a single direction of movement. Each session consisted of a practice block, then one block of experimental trials under no-perturbation conditions and three blocks of 80 trials each in which $10 \mathrm{~mm}$ displacements were interspersed in $20 \%$ of trials. The no-perturbation block was used to calculate the mean trajectory and the AR model of trial-to-trial variation in position, velocity and force signals. The task was designed such that the servo-displacement occurred at the same position in the center of the workspace as that used to estimate stiffness during static conditions. Specifically, the servo-displacement was initiated 10 $\mathrm{cm}$ from the start of movement. As in the static condition the servo was ramped on and off over $100 \mathrm{~ms}$ and there was a $200 \mathrm{~ms}$ hold phase. The final $50 \mathrm{~ms}$ of hold phase was 
centered at the point used for stiffness estimation under static conditions. Before and after the position-servo interval, the hand moved freely and no perturbations or force due to robot were applied in any way. The actual position of the hand in the middle of measurement interval averaged $[-0.004 \pm 0.001 \mathrm{~m}, 0.0247 \pm 0.003 \mathrm{~m}]$ in robot coordinates. Tests for stiffness under static conditions were conducted with a target position at $[0$, $0.025 \mathrm{~m}]$.

Measures of displacement and restoring force that were used for stiffness estimation were obtained in a $50 \mathrm{~ms}$ window starting $250 \mathrm{~ms}$ following the onset of the position-servo. Figure 2 gives representative examples of variation during this interval in position and force measurements (mean $\pm 1 \mathrm{SD}$ ) during longitudinal movements in which lateral perturbations are applied in both directions. To assess the possibility of voluntary intervention, we examined variability in position, velocity, and restoring force during the $50 \mathrm{~ms}$ interval used for stiffness estimation. The assumption was that voluntary intervention during the measurement interval would be reflected in an increase in variation due to the subject's response to the servo position displacement. For purposes of comparison, we first examined variability during the interval in no-perturbation movements that corresponded to the period of servo-displacement during perturbation trials. During this interval, the mean change in position, velocity and force (as reflected by the average of standard deviations) was $4.4 \mathrm{~mm}, 2.9 \mathrm{~mm} / \mathrm{s}$ and $0.1 \mathrm{~N}$, respectively (averaged over movements in $\mathrm{X}$ and $\mathrm{Y}$ ). When the limb was displaced under servocontrol, average changes in position, velocity and force during the same interval were 4.5 
$\mathrm{mm}, 3.7 \mathrm{~mm} / \mathrm{s}$, and $0.5 \mathrm{~N}$. Examination of these values thus gives little evidence of voluntary intervention.

As a further check against the possibility that subjects may have intervened voluntarily we have also computed the variation in position, velocity and force over an earlier interval, also $50 \mathrm{~ms}$ in duration, which started $200 \mathrm{~ms}$ after the onset of the perturbation. The results are essentially similar to those reported above. Specifically, the average standard deviation of position, velocity and force during the hold phase of the servo displacement was $5.2 \mathrm{~mm}, 3.9 \mathrm{~mm} / \mathrm{s}$ and $0.5 \mathrm{~N}$ respectively. The values during the corresponding interval in movements without perturbations were $5.8 \mathrm{~mm}, 3.1 \mathrm{~mm} / \mathrm{s}$ and $0.08 \mathrm{~N}$ respectively. Thus, variation in position and velocity is no greater during the hold phase of the servo displacement than during control movements in the absence of the position servo. Variation in force is greater but the average value is still small, in the range of $0.5 \mathrm{~N}$. As a final check we also computed estimates of stiffness during the same two $50 \mathrm{~ms}$ intervals. These two intervals comprised the last $100 \mathrm{~ms}$ of the plateau phase of position servo control. We obtained the following stiffness matrices, averaged over subjects, for these intervals: [-208.2 60.0; 39.2 -47.2] for the earlier interval and [-224.0 $66.8 ; 22.6-24.5]$ for the later interval. It can be seen that the stiffness estimates are quite similar. The similarity argues against the possibility of voluntary intervention and also against the possibility that there has been a change in equilibrium position (even involuntary) as a result of the perturbation. These sorts of changes should result in an increase in restoring force and hence stiffness in the first case and a decrease in the 
second. Overall, there was little evidence of voluntary intervention during stiffness estimation.

Data analysis. Position servo-control during movement requires an accurate prediction of what the hand path would be if the servo was inactive. The algorithm described below generates a predicted movement relative to which the servo is applied. Our method for trajectory estimation is as follows. Each movement under no-perturbation conditions can be expressed as:

$$
X_{i}(n)=\bar{X}(n)+\Delta x_{i}(n)
$$

Where $n$ is the sample number and $X_{i}$ is the current trajectory, $\bar{X}$ is the mean trajectory of trials in the no-perturbation block, and $\Delta x_{i}$ is the variation about the mean. Trial-to-trial variation is represented using an AR model such that Equation 1 can be re-written as:

$$
X_{i}(n)=\bar{X}(n)+\Delta x_{i}(n)=\bar{X}(n)+\sum_{j=1}^{N} a_{j} * \Delta x_{i}(n-j)+\eta_{i}
$$

where $\Delta x_{i}(n-j)$ is the difference between the ith trajectory and the mean trajectory, over the previous $\mathrm{j}$ samples, $\mathrm{a}_{\mathrm{j}} \mathrm{s}$ are AR model coefficients, $\mathrm{N}$ is the AR model order and $\eta_{i}$ is noise corresponding to the unmodeled part of the signal. Eighth-order AR models were used to model the trial-to-trial variation around the mean trajectory of position, velocity and force signals in both $\mathrm{X}$ and $\mathrm{Y}$. A standard linear least-squares method was used to calculate the AR coefficients based upon the block of 80 trials in the noperturbation condition. A $600 \mathrm{~ms}$ interval from each no-perturbation trial was used for purposes of this calculation. The interval began $200 \mathrm{~ms}$ before the point at which the 
perturbation would have been initiated in servo-displacement trials and extended through to $400 \mathrm{~ms}$ beyond this point.

Hand position, velocity and force were sampled at $600 \mathrm{~Hz}$. The force from the robot motors was controlled at the same frequency. Each session began by recording a single block of 80 trials in the absence of servo-displacements. The data from these trials were used to calculate the mean trajectory and also to generate an AR model of trial-totrial variation. Position and velocity signals were down-sampled for AR modeling, such that an eighth-order model estimated the effect of the previous $100 \mathrm{~ms}$ on subsequent samples.

Figure 3 shows an example of prediction error resulting from the use of the mean trajectory of previous trials (dashes) and the AR model of trial-to-trial variation in combination with the mean trajectory (solid) for representative reaching movements in $\mathrm{Y}$. The figure shows a representative trial starting $200 \mathrm{~ms}$ before the onset of the positionservo and continuing throughout a $400 \mathrm{~ms}$ period of servo-control in which the difference between the actual and predicted trajectory is given. Variation in the function shown in dashes reflects the fact that the mean of previous trials differs from the trajectory that is used for present trial. It can be seen that the prediction error for the AR model is small in both $\mathrm{X}$ and $\mathrm{Y}$ position and also for velocity. The prediction error was quantified using a set of 80 movements from the no-perturbation condition in the $\mathrm{Y}$ direction. For this set of movements we evaluated the mean error over the first $300 \mathrm{~ms}$ of the prediction interval. This is the same interval over which the position servo is applied for purposes of stiffness 
estimation during measurement trials. For the AR model, the mean $( \pm \mathrm{SD})$ of the absolute prediction error was $1.2 \pm 0.8$ and $1.3 \pm 0.2 \mathrm{~mm}$ for $\mathrm{X}$ and $\mathrm{Y}$ respectively. The same calculation for velocity in $\mathrm{X}$ and $\mathrm{Y}$ yielded absolute prediction errors of $2.0 \pm 1.7$ and $3.4 \pm 2.1 \mathrm{~mm} / \mathrm{s}$, respectively. This shows that prediction error is comparable in the direction of movement and in the perpendicular direction. When the same calculation was repeated using the mean trajectory, the mean of the absolute positional error was $4.0 \pm 2.5$ and $6.6 \pm 4.8 \mathrm{~mm}$ in $\mathrm{X}$ and $\mathrm{Y}$ respectively. The mean of absolute velocity errors was 9.4 \pm 5.1 and $18.6 \pm 12.1 \mathrm{~mm} / \mathrm{s}$ respectively. Thus, the AR model results in a substantially better estimate of the trajectory for purposes of position servo-control. A further advantage of the AR method is that it minimizes possibly large transient perturbations at the onset of load. Whereas the prediction error for the AR method increases gradually over the prediction interval to reach a maximum towards the end of the interval (see Figure 3), if the mean trajectory was used to predict ongoing movement, there could be large differences between the actual and mean trajectory even at the beginning of the predicted interval (Figure 3) which would result in large transient loads.

In the data analysis, trials were dropped if the difference between the desired and actual position or the change in restoring force in final $50 \mathrm{~ms}$ of the plateau phase of the servo-displacement exceeded $3 \mathrm{~mm}$ or $0.5 \mathrm{~N}$ in either $\mathrm{X}$ or $\mathrm{Y}$. This resulted in rejection of less than $4 \%$ of trials.

The equation of arm motion in planar movement can be expressed as:

$$
I(q) \ddot{q}+C(\dot{q}, q)=\tau(\dot{q}, q, t)+\tau_{e x t}
$$


where $\mathrm{q}$ represents angular position of the shoulder and elbow. The left side of the equation gives the passive dynamics of arm, $I$ is inertia and $C$ represents the Coriolis and centrifugal component. $\tau$ is the torque generated by arm muscles and $\tau_{\text {ext }}$ is external torque due to the robot. For small deviations about the equilibrium trajectory, the equation can be linearized as:

$$
I \delta \ddot{q}+\frac{\partial I \ddot{q}}{\partial q} \delta q+\frac{\partial C}{\partial \dot{q}} \delta \ddot{q}+\frac{\partial C}{\partial q} \delta q=\frac{\partial \tau}{\partial \dot{q}} \delta \dot{q}+\frac{\partial \tau}{\partial q} \delta q+\delta \tau_{e x t}
$$

In the case of position servo-control:

$$
\delta \ddot{q}=\delta \ddot{q} \equiv 0
$$

thus Equation 4 can be rewritten as:

$$
\left(\frac{\partial I \ddot{q}}{\partial q}+\frac{\partial C}{\partial q}\right) \delta q=\frac{\partial \tau}{\partial q} \delta q+\delta \tau_{e x t}
$$

The term $R=-\frac{\partial \tau}{\partial q}$ is the arm stiffness matrix so:

$$
R \delta q+\left(\frac{\partial I \ddot{q}}{\partial q}+\frac{\partial C}{\partial q}\right) \delta q=\delta \tau_{e x t}
$$

Equation 7 can be transformed into hand coordinates by using the Jacobian matrix, $J$, such that:

$$
\begin{aligned}
& \delta q=J^{-1} \delta X \\
& \delta \tau_{e x t}=J^{T} \delta F_{e x t}
\end{aligned}
$$


where $X$ is the position of the hand in Cartesian space and $F_{\text {ext }}$ is the force at the hand due to the robot. After algebraic manipulation, Equation 7 can be re-written as:

$$
\begin{aligned}
& K \delta X+\frac{\partial F_{d}}{\partial q} \delta q=\delta F_{e x t} \\
& K=J^{-T} R J^{-1}, \quad \frac{\partial F_{d}}{\partial q}=J^{-T}\left(\frac{\partial I \ddot{q}}{\partial q}+\frac{\partial C}{\partial q}\right)
\end{aligned}
$$

where $K$ is the hand stiffness matrix, $\delta X$ and $\delta F_{e x t}$ are the change in hand position and force, respectively. As indicated in Equation 9, the restoring force resulting from servodisplacement contains two terms. The first is the force due to limb stiffness and the second is the contribution of dynamics to restoring forces, $\frac{\partial F_{d}}{\partial q}$. We used a numerical method introduced by Burdet and Osu (1999) to calculate the portion of the restoring force due to stiffness of the arm. $\frac{\partial F_{d}}{\partial q}$ is a two-by-two matrix and each element of this matrix can be calculated by using the Equation 10 .

$$
\frac{\partial F_{d, i}}{\partial q_{j}} \equiv \frac{\left(J_{q_{n+1}^{-T}}^{T}\left(I_{n+1} \ddot{q}_{n+1}+C_{n+1}\right)-J_{q_{n}}^{-T}\left(I_{n} \ddot{q}_{n}+C_{n}\right)\right)_{i}}{q_{j, n+1}-q_{j, n}}
$$

In this equation $i$ and $j$ are the $i$ th and $j$ th elements of $F_{d}$ and $q$ vectors, respectively and $n$ is the sample number. Estimates of inertia, $I$, and Coriolis force, $C$, where obtained on a per subject basis using subject height and weight (Winter, 1990).

Simulations. The simulation studies used a model of planar two-joint arm movement based on the $\lambda$-version of the equilibrium point hypothesis (Feldman 1986). In the model, both muscle coactivation and movement are dependent upon the difference between 
actual muscle length, $l$, and a centrally-specified threshold length $(\lambda)$ for motor neuron activation. Muscle activation is given by the following equation

$$
A=l-\lambda+\mu \frac{d l}{d t}
$$

in which $A$ is positive or zero, $\mu$ is a constant specifying the dependence of the activation threshold on the velocity of muscle lengthening or shortening, and $\frac{d l}{d t}$ is velocity dependent afferent feedback. Muscle force, $F$, depends upon muscle activation according to the exponential relationship

$$
F=\rho[\exp (A)-1]
$$

where force varies in proportion to the physiological cross sectional area, $\rho$, of each modeled muscle (Winters and Woo, 1990). The model includes single-joint muscles at the shoulder and elbow and two-joint muscles that span both joints. Musculo-skeletal geometry is estimated from anatomical sources (An et al. 1981, 1989; Winters and Woo 1990). The muscle model is a variant of the Zajac (1989) formulation and includes the dependence of force on length and velocity and also excitation and contraction dynamics and passive muscle stiffness. Length and velocity dependent reflex delays are also modeled. Extensor muscle moment arm are assumed to be constant for all three extensor muscles $(2 \mathrm{~cm}$ at the elbow and $4 \mathrm{~cm}$ at the shoulder). Flexor moment arms vary with joint angle and are calculated on a geometrical basis (values range between 2.5 to $5 \mathrm{~cm}$ ). The inertia and geometrical constants of the upper and lower arm are: upper and lower arm mass: 2.1 and $1.65 \mathrm{~kg}$, length: 0.34 and $0.46 \mathrm{~cm}$, and moment of inertia about the center of mass: 0.015 and $0.022 \mathrm{~kg} \cdot \mathrm{m}^{2}$. The modeled physiological cross-sectional area, obtained from Winters and Woo (1990), was $2.1 \mathrm{~cm}^{2}$ for biceps short head; $11 \mathrm{~cm}^{2}$ for 
biceps long head; $14.9 \mathrm{~cm}^{2}$ for deltoid; $14.9 \mathrm{~cm}^{2}$ for pectoralis; $12.1 \mathrm{~cm}^{2}$ for triceps lateral head and $6.7 \mathrm{~cm}^{2}$ for triceps long head. See Gribble et al. (1998) for a more detailed presentation of the model.

In the simulations presented below, separate commands analogous to the $\mathrm{R}$ and $\mathrm{C}$ commands of the one joint model are defined to produce movement and muscle coactivation respectively. Movements are generated assuming straight line equilibrium shifts in hand space. Because the number of modeled muscles exceeds the number of kinematic degrees of freedom, there are, in principle, an infinite number of sets of $\lambda$ shifts that correspond to each point along the equilibrium trajectory, where each set results in a different level of muscle force. Analogous to the R command in the one joint model, we assume that movements at each point on the trajectory arise as a result of the set of $\lambda \mathrm{s}$ that minimizes total muscle force.

Consistent with experimental evidence (Gribble and Ostry, 1998, Gomi and Osu, 1998), we defined separate cocontraction commands at the shoulder and the elbow. Cocontraction at the shoulder was specified by the set of $\lambda$ shifts that increased shoulder muscle forces without changing net shoulder torque. Similarly, the elbow concontraction command increased elbow and double joint muscle forces without changing net elbow torque. As in Gribble et al. (1998), for purposes of these simulations, the cocontraction commands were defined initially in force space, and hence the units of the cocontraction command are $\mathrm{N}$ and specify average muscle force. The vector in $\lambda$ space associated with this change in muscle force in statics was used as the cocontraction command. 


\section{RESULTS}

To verify the performance of the trajectory predictor, a block of eighty trials in the $\mathrm{Y}$ direction was recorded during which a position-servo of zero amplitude was applied. With an ideal predictor the forces sent to the robot should be zero (since actual and predicted trajectories are the same). During this test block, mean forces sent to the robot in $\mathrm{X}$ and $\mathrm{Y}$ were $-0.07 \mathrm{~N}$ and $\approx 0 \mathrm{~N}$ and the mean commanded tangential force was $0.83 \mathrm{~N}$. The prediction error was thus centered about zero and the absolute error in prediction was small. We further examined our trajectory prediction algorithm by comparing mean position, velocity and force trajectories for a position servo of zero amplitude with other trajectories drawn from no-perturbation movements. In each case we used values that extended from $200 \mathrm{~ms}$ before the onset of the position servo through to the end of the prediction window. Correlation coefficients between the zero amplitude servo and no-perturbation movements were 0.96 and 0.99 for position signals in $\mathrm{X}$ and $\mathrm{Y}$. The results for velocity were 0.94 and 0.99 and for force, 0.91 and 0.99 . These results assured us that the method of trajectory prediction was capable of closely replicating the unperturbed movement.

Figure 4 shows an example of servo-displacements during longitudinal movements. Panel A shows servo-displacements in $\mathrm{Y}$ (along the axis of movement); Panel B gives displacements in X. It can be seen that the $50 \mathrm{~ms}$ that is used for stiffness estimation is centered in the middle of movements. In Panel A, the plateau phase of the 
displacement in Y shows no effect of servo-control on movement in $\mathrm{X}$ nor on velocity in $\mathrm{X}$ or $\mathrm{Y}$. Following the completion of the position-servo a deviation of the hand can be seen in the $\mathrm{X}$ direction. During this interval, the maximum departure in $\mathrm{X}$ from the mean trajectory was $0.5 \mathrm{~cm}$ for position and $2.7 \mathrm{~cm} / \mathrm{s}$ for velocity. The outlined portion in Panel $\mathrm{A}$ is shown in Panel $\mathrm{C}$ as a difference score relative to the no-perturbation trajectory. As can be seen, the subject's hand was displaced about $10 \mathrm{~mm}$ and there is no resonance during the plateau phase. Figure 4D shows hand paths in the horizontal plane when the servo-displacement is in $\mathrm{Y}$.

Figure 4B shows servo-displacements in $\mathrm{X}$. There is no effect of the positionservo on movement in Y (neither in position nor velocity). The plateau phase of positionservo in $\mathrm{X}$ is stable and without resonance. Figure 4E shows there is no departure from the no-perturbation trajectory in the $\mathrm{Y}$ direction when position-servo is in $\mathrm{X}$. Figure $4 \mathrm{~F}$ shows the hand path in the horizontal plane for servo displacements in $\mathrm{X}$. The information presented in Figure 4 thus shows that the servo can be applied in each direction without affecting position or velocity in the other direction.

Figure 5A shows hand stiffness ellipses under stationary conditions and during movement in $\mathrm{X}$ and $\mathrm{Y}$. The solid lines show the static condition, dots are for movements in $\mathrm{Y}$ and dashes for movements in $\mathrm{X}$. The mean orientation of the major axis of the ellipse relative to the horizontal axis was $123.8 \pm 11.1,130.4 \pm 12.6$ and $164.5 \pm 9.8 \mathrm{deg}$ for the static condition, and for movement in $\mathrm{X}$ and $\mathrm{Y}$ respectively. Differences in orientation were assessed using repeated-measures ANOVA followed by Bonferroni 
corrected post-hoc tests. In comparison to static conditions, there was a significant counter-clockwise rotation of ellipse during movement in $\mathrm{Y}(\mathrm{p}<0.01)$. The orientation of the ellipse for movement in $\mathrm{X}$ was not different than that under static conditions $(\mathrm{p}>$ 0.05). We assessed differences in the size and the shape of the stiffness ellipse. The shape of the ellipse, as defined by ratio of the major to minor axis, was $2.5 \pm 0.7,2.62 \pm 1.0$ and $10.6 \pm 2.9$ for statics, $\mathrm{X}$ and $\mathrm{Y}$ respectively. The shape changed reliably for movement in $\mathrm{Y}$ such that it was thinner than in the two other conditions $(\mathrm{p}<0.01)$. There were no reliable differences in the size of the ellipse as defined by the enclosed area $(p>0.05)$. Values of $2.2 \pm 0.8,3.4 \pm 1.4$ and $1.9 \pm 1.0 \mathrm{~N}^{2} / \mathrm{cm}^{2}$ were obtained for this measure. Table 1A shows the numerical value of stiffness matrices for all subjects and conditions. Table 1B gives the corresponding values in joint coordinates.

We assessed the statistical reliability of the anti-symmetric term of the joint stiffness matrix by using, as elsewhere, between subjects ANOVA. The anti-symmetric term is the mean of the difference between the two off-diagonal terms. It has been suggested that non-zero anti-symmetric values result from an imbalance in reflex interactions between shoulder and elbow muscles (Hogan, 1985). We found that the antisymmetric term of the joint stiffness matrix differed in magnitude depending on movement direction $(\mathrm{p}<0.05)$ and was reliably different than zero for movements in $\mathrm{Y}$ $(\mathrm{p}<0.05)$. The anti-symmetric term was not found to be reliably different than zero for movements in $\mathrm{X}$ or for measures under postural maintenance conditions. 
Simulation studies. To understand the empirically observed patterns, we simulated the stiffness that might be expected by using a two-joint planar arm model described in Gribble et al. (1998). The simulations replicated the procedures in the experimental study. Modeled control signals were based on the $\lambda$ version of equilibrium-point hypothesis. Commands analogous to $\mathrm{R}$ and $\mathrm{C}$ commands in previous versions of the model (Gribble et al., 1998) were used to produce rotation of the joints and muscle coactivation without movement. To obtain estimates of stiffness in statics, values for $\mathrm{R}$ and $\mathrm{C}$ commands were held constant to simulate postural maintenance. To obtain estimates of stiffness during movement, the $\mathrm{R}$ command was varied to simulate movement. Separate cocontraction commands were defined for the elbow and shoulder. The elbow cocontraction command was applied to single-joint elbow muscles and biarticular muscles (Gribble and Ostry, 1998; Gomi and Osu, 1998). The shoulder cocontraction command was applied to single-joint shoulder muscles.

Figure 5B shows the simulated ellipses for the conditions tested experimentally. The corresponding stiffness matrices are given in Table 2 in both hand and joint coordinates. The left, center and right panels of Figure 5B give different strategies for stiffness regulation. To simulate the stiffness ellipse under static conditions, shoulder and elbow cocontraction commands were set to $15 \mathrm{~N}$ and values associated with the $\mathrm{R}$ command were held constant. The resulting ellipse is shown with a solid line in all three panels. The orientation of the major axis of this ellipse is 121.0 deg relative to the horizontal and its size is $1.95 \mathrm{~N}^{2} / \mathrm{cm}^{2}$ which is comparable to that observed in the empirical data. Simulated stiffness ellipses for movements in $\mathrm{Y}$ are shown with dots and 
for $\mathrm{X}$ with dashes. The results presented in the three panels were obtained using different cocontraction commands. In the left panel, shoulder and elbow commands during movement where unchanged from those under static conditions ( $15 \mathrm{~N}$ each). In the center panel, shoulder and elbow commands were both increased to $30 \mathrm{~N}$. In the right panel, for movements in $\mathrm{Y}$, elbow cocontraction was constant at $10 \mathrm{~N}$ and shoulder cocontraction was set to $60 \mathrm{~N}$. For movements in $\mathrm{X}$, shoulder was constant at $20 \mathrm{~N}$ and elbow was increased to $50 \mathrm{~N}$. For all cocontraction strategies (including the left panel where cocontraction was unchanged from static conditions), the stiffness ellipse rotated counterclockwise during movement in $\mathrm{Y}$ and rotated clockwise by a lesser amount for movement in $\mathrm{X}$. The shape and orientation of the actual ellipse for movement in $\mathrm{Y}$ is closely matched by the ellipse in the right panel whereas, for movement in $\mathrm{X}$, the pattern shown in the center panel is closest to that observed empirically. The orientation of the simulated ellipse for movements in $\mathrm{Y}$ is 144.6, 142.4 and $145.1 \mathrm{deg}$, the orientation for $\mathrm{X}$ is $120.2,119.2$ and $109.9 \mathrm{deg}$, for left to right panels respectively.

Figure 6 shows stiffness ellipses in joint coordinates. The solid line gives joint stiffness under static conditions. The dotted line is for movements in $\mathrm{Y}$, the dashed line is for movements in X. For purposes of graphical presentation of the empirical data, we averaged the joint stiffness matrices of the six experimental subjects. Figure $6 \mathrm{~A}$ gives the associated joint stiffness ellipses. Figures 6B - D show simulated joint stiffness ellipses. Figure $6 \mathrm{~B}$ shows joint stiffness under static conditions and for movements in $\mathrm{X}$ and $\mathrm{Y}$. For both posture and movement, the modeled cocontraction command is $15 \mathrm{~N}$ at the shoulder and elbow. Figure $6 \mathrm{C}$ shows the effects of using a cocontraction command of 
$30 \mathrm{~N}$ during movement while hold cocontraction under static conditions to $15 \mathrm{~N}$. Figure $6 \mathrm{D}$ shows the effects of different levels of cocontraction on movements in $\mathrm{X}$ and $\mathrm{Y}$. For the $\mathrm{Y}$ direction, $10 \mathrm{~N}$ and $60 \mathrm{~N}$ are used at the elbow and shoulder respectively. For the $\mathrm{X}$ direction, $50 \mathrm{~N}$ and $20 \mathrm{~N}$ are used at the elbow and shoulder. Stiffness in statics is simulated using $15 \mathrm{~N}$ cocontraction at both joints. It should be noted that there is a close correspondence between the joint stiffness ellipses based on empirical data and those obtained from simulations. As in the hand stiffness stimulations shown in Figure 5, the ellipse for the actual movement in $\mathrm{Y}$ is matched by the ellipse shown in Figure 6D where shoulder stiffness is $60 \mathrm{~N}$ and elbow is $10 \mathrm{~N}$. Similarly, the empirical data for movement in $\mathrm{X}$ is closest to the corresponding ellipse in Figure 6C (30N at each of the shoulder and elbow).

We used the model to assess stiffness during postural maintenance at muscle activation levels that were matched to those obtained during movement. The goal was to determine if there is something unique about the changing patterns of muscle activation during movement or simply the fact that muscle activation at levels matched to those in movement results in the change in modeled stiffness ellipse orientation. The modeled control signals needed to match muscle activation levels were obtained by selecting from simulated movements the set of muscle $\lambda$ s associated with the hold phase of the perturbation - the point at which stiffness was estimated. This set of $\lambda \mathrm{s}$ was used in combination with modeled servo displacements to obtain estimates of stiffness, with the limb fixed at the center of the workspace by simulating a high stiffness robot handle at the desired measurement point. The observed patterns of hand stiffness are shown in 
Figure 7. It can be seen that when muscle activation levels are matched to those observed in movement, the orientation of the simulated stiffness ellipses tends to match those observed empirically. Stiffness at muscle activation levels equal to those in statics is shown as a solid line. When $\lambda \mathrm{s}$ are chosen to match activation during longitudinal movements (dots), the simulated stiffness ellipse rotates counter-clockwise, as is observed empirically. Similarly, when $\lambda$ s produce activation that is matched to lateral movements (dashes), small clockwise changes in orientation are observed. These observations are consistent with the idea that differences in stiffness ellipse orientation under static conditions and during movement arise as a consequence of the patterns of muscle activation that drive the limb.

\section{DISCUSSION}

A limited ability for stiffness change has been observed under static conditions (Gomi and Osu 1998; Perreault et al. 2002; Darainy et al. 2004) whereas the capacity for stiffness control during reaching movements appears to be more substantial (Burdet et al., 2001; Franklin et al., 2003). In the present paper we have tried to assess some of the reasons for this discrepancy.

As a first step in understanding this difference, we have evaluated the degree to which stiffness changes from posture to movement even when there are no destabilizing forces involved. We have estimated arm stiffness in the center of the workspace during postural maintenance and in the same central location during lateral and longitudinal 
movements. During longitudinal movements, we observed a counter-clockwise rotation of the hand stiffness ellipse of almost $40 \mathrm{deg}$ in comparison to its orientation under static conditions. During lateral movements, there was no reliable change in ellipse orientation.

We used a model of planar shoulder and elbow movement to investigate the manner in which control signals to muscles might be altered to produce the empirically observed patterns of stiffness. Two different commands provided for movement and for muscle cocontraction - one that shifts the position of the limb at a fixed level of cocontraction (analogous to the R command in a single-joint model), the other that results in changes in muscle coactivation without any movement (analogous to the $\mathrm{C}$ command). In the simulation studies, when we used the model to compare stiffness estimates during movement and in statics, we found that even when the magnitude of the cocontraction command was the same in statics and during movement (Figure 5B left panel), the orientation of the stiffness ellipse shifted in a manner similar to that observed empirically, both for lateral and longitudinal movement directions. In effect, the same centrally specified coactivation level led to different patterns of force opposing servo-displacement when combined with different commands that result in movement.

An examination of the simulation results (Figures 5B and 7) shows that the main determinant of the orientation of the stiffness ellipse in our modeling studies is the pattern of muscle activation during movement and also movement direction. Changes in the overall cocontraction level and in the balance of cocontraction between the elbow and the shoulder have noticeable but smaller effects on ellipse orientation. The simulations show 
that different overall levels of cocontraction primarily produce changes in the size and shape of the ellipse (Figure 5B middle panel). Changes in the balance of shoulder and elbow cocontraction can also affect the orientation of the ellipse as shown for movements in X (right panel).

The anti-symmetric term of the joint stiffness matrix was found to be reliably different than zero for movements in the longitudinal direction. This finding is relevant to a proposal by Hogan (1985) that the asymmetry arises from inter-joint reflexes that are unequal in magnitude. The basic idea here is as follows. The off-diagonal terms of the joint stiffness matrix give the torque at the shoulder due to motion at the elbow and also torque at the elbow due to motion of the shoulder. Inter-joint reflexes contribute to the torque that arises at one joint as a consequence of movement at another. When inter-joint reflex gains are equal in magnitude then, for the same displacements at the shoulder and elbow, the reflex contribution to the restoring force at the opposite joint will be equal torque change at the elbow due to shoulder motion will tend to be offset by torque change at the shoulder due to elbow motion. Any asymmetry in inter-joint reflex gains could lead to differences in the magnitude of these off-diagonal elements and consequently to asymmetric stiffness matrices. It should be noted, that there are other factors in addition to an asymmetry in inter-joint reflex gains that could contribute to an imbalance in the magnitude of the off-diagonal terms. In particular, differences in muscle moment arm lengths at the shoulder and the elbow in combination with a non-linear dependence of force on muscle length, mean that the same change in joint angle at the shoulder and elbow can result in different torques at the two joints. 
It is informative in this context to consider the results of our modeling studies. The model in its present form does not include inter-muscular reflex interactions. It does however model the non-linear dependence of force on muscle length and also variation in muscle moment arm lengths. As shown in Table 2, the simulated stiffness matrices are nearly symmetric. This could indicate that asymmetry in stiffness that is observed empirically arises more as a result of reflex interaction, which is not modeled, than nonlinearities in muscle mechanical behavior, which are.

A number of recent studies have reported arm stiffness in the absence of external load during reaching movements along lateral or longitudinal axes (Gomi and Kawato, 1997, Burdet et al., 2001; Mah, 2001; Frolov et al., 2006). The studies use two different approaches for stiffness estimation, one using open-loop force control (Gomi and Kawato, 1997, Mah, 2001; Frolov et al., 2006), and the other, which includes the present paper, use position-servo control (Burdet et al., 2001). For movements in a lateral direction, the present paper and those of Gomi and Kawato (1997) and Frolov et al. (2006) find that movement alone produces little systematic change in the orientation of the hand stiffness ellipse relative to that observed under postural conditions. In contrast, for movements in a longitudinal direction a broad range of hand stiffness ellipse orientations has been observed. Gomi and Kawato (1997) report an ellipse orientation that rotates clockwise relative to that observed under static conditions. In contrast, Frolov et al. (2006) report a large counterclockwise rotation of the stiffness ellipse. Indeed, the orientation of the ellipse reported by Frolov et al. (2006), in the absence of external load, 
is comparable to that described by Burdet et al. (2001) after learning a divergent force field. The present paper likewise documents a substantial counterclockwise rotation relative to statics, but one that is clearly less than that found in Frolov et al. and quite close in value (within about $10 \mathrm{deg}$ ) to that obtained by Burdet et al. (2001).

Studies by Frolov et al. (2006) and by Gomi and Kawato (1997) differ in several ways from those reported here and the differences in procedure and in the direction of movement may well account for the observed differences in stiffness ellipse orientation. First, the direction of movement differed in Gomi and Kawato (1997) and Frolov et al. (2006). Frolov et al. used near to far movements; Gomi and Kawato used far to near. Moreover, Frolov et al. (2006) used force pulses and a restricted set of force application directions. Perturbations were delivered in three directions only that were separated by 60 deg. Gomi and Kawato (1997) also used force pulses and, as a reference trajectory, they used a mean of unperturbed movements. Their procedure also required estimation of inertia, viscosity and stiffness. These differences in both movement direction and procedure could have contributed to the different estimates of stiffness ellipse orientation.

The simulated and empirical values of joint stiffness in the present paper are comparable to those reported in other studies. Estimates of static joint stiffness from Tsuji et al. (1995) and Gomi and Kawato (1997) range from 5 to 20 N.m/rad for shoulder stiffness, 4 to $13 \mathrm{~N} . \mathrm{m} / \mathrm{rad}$ for elbow stiffness and 1 to $7 \mathrm{~N} . \mathrm{m} / \mathrm{rad}$ for the off-diagonal terms of the joint stiffness matrix. Our empirical and modeled values are both within this range. Our estimates of joint stiffness during movement are for the most part comparable 
to those reported by others. Gomi and Kawato (1997) and Frolov et al. (2006) report peak values for shoulder stiffness of about $35 \mathrm{~N} . \mathrm{m} / \mathrm{rad}$, for elbow stiffness of $20 \mathrm{Nm} . \mathrm{rad}$ and about 10 N.m/rad for the off-diagonals. Our modeling results are similar to these. Our empirical estimates, particularly for movement in the Y direction, are somewhat larger, in some cases by as much as a factor of two.

In studies to date on impedance control in adaptation to destabilizing force-fields, stiffness has been estimated during reaching in the longitudinal direction. There are no reports of stiffness control in conjunction with lateral reaching movements in divergent force-fields. Indeed the only studies that have measured the arm stiffness during lateral movements have done so under no-perturbation field conditions (Gomi and Kawato, 1997, Mah, 2001; Frolov et al., 2006). Since the orientation of the stiffness ellipse during lateral movements is typically close to the longitudinal axis, one would expect little change in orientation during lateral movements in the presence of destabilizing loads because there is already ample stiffness in this direction. 


\section{ACKNOWLEDGEMENTS}

We thank G. Houle for advice and assistance. This research was supported by the National Institute of Child Health and Human Development grant HD-48924, the Natural Sciences and Engineering Research Council of Canada, and Fonds québécois de la recherche sur la nature et les technologies. 


\section{REFERENCES}

An K, Hui F, Morrey B, Linscheid R, Chao E. Muscles across the elbow joint: a biomechanical analysis. J. Biomech. 14: 659-669, 1981.

An K, Kaufman K, Chao E. Physiological considerations of muscle force through the elbow joint. J. Biomech. 22: 1249-1256, 1989.

Burdet E, Osu R, Franklin DW, Milner TE, and Kawato M. The central nervous system stabilizes unstable dynamics by learning optimal impedance. Nature 414: 446-49, 2001.

Burdet E, Osu R, Franklin DW, Yoshioka T, Milner TE, and Kawato M. A method for measuring endpoint stiffness during multi-joint arm movements. J Biomech 33: 1705-09, 2000.

Burdet E and Osu R. Development of a new method for identifying muscle stiffness during human arm movements. Report 1-21, Kawato dynamic brain project, ERATO, Japan.

Darainy M, Malfait N, Gribble PL, Towhidkhoh F, and Ostry DJ Learning to control arm stiffness under static conditions. J Neurophysiol 92: 3344-50, 2004. 
Franklin DW, Burdet E, Osu R, Kawato M, and Milner TE. Functional significance of stiffness in adaptation of multijoint arm movements to stable and unstable dynamics. Exp Brain Res 151: 145-57, 2003.

Frolov AA, Prokopenko RA, Dufosse M, and Ouezdou FB. Adjustment of the human arm viscoelastic properties to the direction of reaching. Biol Cybern 94: 97-109, 2006.

Gomi $\mathrm{H}$ and Kawato M. Human arm stiffness and equilibrium point trajectory during multijoint movement. Biol. Cybern. 76: 163-71, 1997.

Gomi $\mathrm{H}$ and Osu R. Task dependent viscoelasticity of human multijoint arm and its spatial characteristic for interaction with environment. J Neurosci 18: 8965-8978, 1998.

Gribble PL and Ostry DJ. Independent coactivation of shoulder and elbow muscles. Exp Brain Res 123: 355-360, 1998.

Gribble PL, Ostry DJ, Sanguineti V, and Laboissière R. Are complex control signals required for human arm movement? J Neurophysiol 79: 1409-1424, 1998.

Hogan N. The mechanics of multijoint posture and movement control. Biol Cybern 52: 315-331, 1985.

Mah CD. Spatial and temporal modulation of joint stiffness during multijoint movement. 
Exp Brain Res 136: 492-506, 2001.

Perreault EJ, Kirsch RF, and Crago PE. Voluntary control of static endpoint stiffness during force regulation tasks. J Neurophysiol 87: 2808-2816, 2002.

Tsuji T, Morasso PG, Goto K, Ito K. Human hand impedance characteristics during maintained posture. Biol Cybern. 72:475-85, 1995.

Winter DA. Biomechanics and Motor Control of Human Movement, Second Edition. New York: John Wiley \& Sons, 1990.

Winters J, Woo SY. (Eds.) Multiple Muscle Systems: Biomechanics and Movement Organization. New York: Springer-Verlag, 1990.

Zajac F. Muscle and tendon: properties, models, scaling, and application to biomechanics and motor control. CRC Crit. Rev. Biomed. Eng. 17: 359-415, 1989. 


\section{FIGURE LEGENDS}

Figure 1. Experimental setup. Hand stiffness was measured at the center of workspace under static conditions and during longitudinal and lateral movements.

Figure 2. Representative example of position servo displacement and measured restoring force. The movement was in the longitudinal direction $(\mathrm{Y})$; the position servo acted orthogonal to the direction of movement (perturbations in opposing lateral directions are shown in the left and right panels). The vertical grey bar indicates the interval during which estimates of stiffness were obtained.

Figure 3. Example of prediction errors in position and velocity for two methods of trajectory prediction. The dashes shows prediction error when the mean of last eighty trials is used to predict an ongoing movement. The solid line shows prediction error when the mean in combination with the output of an AR model is used.

Figure 4. Position-servo during longitudinal movements. (A) Mean ( $\pm 1 \mathrm{SD}$ ) of hand movement in the absence of load and with a $1 \mathrm{~cm}$ displacement in $\mathrm{Y} . \mathrm{V}_{\mathrm{x}}$ and $\mathrm{V}_{\mathrm{y}}$ indicate velocity, $\mathrm{P}_{\mathrm{x}}$ and $\mathrm{P}_{\mathrm{y}}$ give position. Apart from the applied displacement in $\mathrm{Y}$, there is little change in either position or velocity relative to no-perturbation movements. (B) Mean ( \pm $1 \mathrm{SD}$ ) of hand movement without load and with a $1 \mathrm{~cm}$ servo-displacement in $\mathrm{X}$. (C) Deviation from mean trajectory in Y direction, during $1 \mathrm{~cm}$ displacement in Y. (D) Hand 
path in horizontal plane. Note that the position-servo has no effect on the lateral direction. (E) Deviation from mean trajectory in the $\mathrm{Y}$ during $1 \mathrm{~cm}$ position-servo in $\mathrm{X}$. There is no effect of the position-servo in $\mathrm{X}$ on movement in $\mathrm{Y}$. (F) Hand path in the horizontal plane during position-servo in $\mathrm{X}$.

Figure 5. Empirical and simulated hand stiffness ellipses. The lateral direction is given on the horizontal; the longitudinal direction is on the vertical. Stiffness measures were taken with the elbow at $90 \mathrm{deg}$ and the shoulder at $45 \mathrm{deg}$. (A) Stiffness ellipses under static conditions and during longitudinal and lateral movements for subjects S1 - S6. The solid line shows the static stiffness ellipse, dots show the ellipse during longitudinal movements. Dashes represents stiffness during lateral movement. (B) Simulated stiffness ellipse under static conditions (solid) and during longitudinal (dots) and lateral (dashes) movements. (Left panel) Elbow and shoulder cocontraction are $15 \mathrm{~N}$ for static stiffness estimates and for lateral and longitudinal movements. (Center panel) Elbow and shoulder cocontraction are $15 \mathrm{~N}$ in statics and $30 \mathrm{~N}$ during movement. (Right panel) Elbow and shoulder cocontraction are $15 \mathrm{~N}$ (statics). For longitudinal movement, shoulder cocontraction is $60 \mathrm{~N}$ while elbow cocontraction is $10 \mathrm{~N}$. For the lateral condition, the shoulder is at $20 \mathrm{~N}$ and the elbow is $50 \mathrm{~N}$.

Figure 6. Empirical and simulated joint stiffness ellipses. Shoulder stiffness is given on the horizontal; elbow stiffness is on the vertical. (A) Empirical joint stiffness ellipses under static conditions (solid) and during reaching movements in $\mathrm{X}$ (dashes) and $\mathrm{Y}$ (dots). The empirical ellipses were derived by averaging stiffness matrices over subjects. 
(B) Simulated joint stiffness ellipses in the same three conditions. The shoulder and elbow cocontraction command were $15 \mathrm{~N}$ in all three conditions. (C) Static joint stiffness was simulated using a $15 \mathrm{~N}$ cocontraction command at both the elbow and the shoulder. $30 \mathrm{~N}$ cocontraction commands were used when stiffness was simulated during movement. (D) For movement in the $\mathrm{Y}$ direction, the shoulder cocontraction command was $60 \mathrm{~N}$ and elbow cocontraction command was $10 \mathrm{~N}$. For movements in $\mathrm{X}$, the shoulder cocontraction command was $20 \mathrm{~N}$ and the elbow cocontraction command was $50 \mathrm{~N}$.

Figure 7. Simulated hand stiffness ellipses in which stiffness is assessed during postural maintenance at the same muscle activation levels as in movement (see text for details). Stiffness at muscle activation levels equal to static conditions is shown as a solid line. Stiffness for muscle activation matched to longitudinal movements is shown with dots. When activation is matched to lateral movements stiffness patterns shown with dashes are obtained. Under static conditions a simulated cocontraction command of $15 \mathrm{~N}$ at both the shoulder and elbow was used. For the longitudinal match, cocontraction levels of 60 $\mathrm{N}$ and $10 \mathrm{~N}$ were used at the shoulder and elbow respectively. For the lateral match, cocontraction was set at $30 \mathrm{~N}$ for both joints. 
A. Hand Stiffness Estimates

\begin{tabular}{|r|rr|rr|rr|}
\hline & \multicolumn{2}{|c|}{ Postural Maintenance } & \multicolumn{2}{c|}{ X direction } & \multicolumn{2}{c|}{ Y direction } \\
\hline S1 & $-68.5 \pm 5.6$ & $30.9 \pm 5.4$ & $-81.1 \pm 13.9$ & $24.1 \pm 9.6$ & $-243.0 \pm 10.5$ & $109.8 \pm 13.5$ \\
& $21.1 \pm 4.8$ & $-138.8 \pm 7.8$ & $35.0 \pm 12.6$ & $-156.9 \pm 8.7$ & $157.8 \pm 13.5$ & $-24.2 \pm 17.4$ \\
\hline S2 & $-103.0 \pm 7.8$ & $38.7 \pm 5.7$ & $-155.8 \pm 9.0$ & $31.5 \pm 7.5$ & $-230.4 \pm 10.0$ & $10.0 \pm 10.2$ \\
& $45.6 \pm 4.9$ & $-79.7 \pm 4.0$ & $63.9 \pm 6.3$ & $-112.6 \pm 5.2$ & $60.6 \pm 5.5$ & $-28.3 \pm 5.6$ \\
\hline S3 & $-89.1 \pm 8.0$ & $18.7 \pm 6.1$ & $-117.1 \pm 25.0$ & $15.0 \pm 15.4$ & $-250.1 \pm 8.2$ & $-2.7 \pm 7.7$ \\
& $28.4 \pm 5.2$ & $-99.5 \pm 5.0$ & $34.4 \pm 10.9$ & $-119.3 \pm 6.7$ & $21.2 \pm 6.8$ & $-21.1 \pm 6.4$ \\
\hline S4 & $-72.3 \pm 6.1$ & $38.6 \pm 3.2$ & $-103.1 \pm 14.1$ & $96.5 \pm 12.2$ & $-223.9 \pm 9.8$ & $11.7 \pm 8.3$ \\
& $39.0 \pm 5.9$ & $-129.3 \pm 6.0$ & $57.9 \pm 9.5$ & $-141.0 \pm 8.2$ & $85.1 \pm 5.8$ & $-40.8 \pm 4.9$ \\
\hline S5 & $-51.9 \pm 5.3$ & $32.5 \pm 4.8$ & $-54.0 \pm 2.8$ & $11.7 \pm 4.4$ & $-175.2 \pm 4.7$ & $-2.4 \pm 4.8$ \\
& $31.6 \pm 2.2$ & $-68.8 \pm 3.3$ & $35.0 \pm 1.9$ & $-57.1 \pm 2.9$ & $16.6 \pm 2.7$ & $-14.8 \pm 2.8$ \\
\hline S6 & $-81.1 \pm 5.7$ & $28.4 \pm 6.5$ & $-126.9 \pm 26.4$ & $73.3 \pm 20.9$ & $-221.3 \pm 12.3$ & $8.9 \pm 11.2$ \\
& $19.6 \pm 6.0$ & $-112.3 \pm 6.1$ & $38.8 \pm 17.4$ & $-122.8 \pm 13.8$ & $59.6 \pm 5.4$ & $-18.0 \pm 4.9$ \\
\hline
\end{tabular}

B. Joint Stiffness Estimates

\begin{tabular}{|l|rl|rr|rr|}
\hline & \multicolumn{2}{|c|}{ Fixed Posture } & \multicolumn{2}{c|}{ X direction } & \multicolumn{2}{c|}{ Y direction } \\
\hline \multirow{2}{*}{ S1 } & -15.20 & -4.48 & -18.01 & -6.70 & -53.65 & -14.94 \\
& -5.58 & -8.98 & -5.48 & -10.34 & -9.56 & 0.02 \\
\hline \multirow{2}{*}{ S2 } & -22.84 & -7.38 & -34.66 & -14.24 & -51.48 & -25.05 \\
& -6.61 & -5.69 & -10.60 & -10.0 & -19.37 & -10.87 \\
\hline \multirow{2}{*}{ S3 } & -19.85 & -8.14 & -26.12 & -11.77 & -56.07 & -28.79 \\
& -7.05 & -8.18 & -9.60 & -10.81 & -26.11 & -14.61 \\
\hline \multirow{2}{*}{ S4 } & -15.97 & -3.99 & -22.63 & -0.89 & -49.93 & -24.09 \\
& -3.95 & -7.17 & -5.22 & -5.18 & -15.86 & -9.70 \\
\hline \multirow{2}{*}{ S5 } & -11.44 & -2.27 & -11.97 & -4.86 & -39.28 & -20.22 \\
& -2.37 & -3.27 & -2.24 & -3.72 & -18.09 & -10.16 \\
\hline \multirow{2}{*}{ S6 } & -18.05 & -6.16 & -28.11 & -6.25 & -49.44 & -24.12 \\
& -7.15 & -8.40 & -10.12 & -7.95 & -18.43 & -9.87 \\
\hline
\end{tabular}

Table 1. Empirical estimates of stiffness $( \pm 95 \% \mathrm{CI})$ during longitudinal and lateral reaching movements and also under static conditions. Panel A gives the hand stiffness matrix $\left\lfloor\begin{array}{l}K_{x x} K_{x y} \\ K_{y x} K_{y y}\end{array}\right\rfloor$ in N/m; B gives the joint stiffness $\left\lfloor\begin{array}{l}R_{s s} R_{s e} \\ R_{e s} R_{e e}\end{array}\right\rfloor$ in N.m/rad. 
A. Simulated Hand Stiffness

\begin{tabular}{|c|c|c|c|}
\hline & Condition 1 & Condition 2 & Condition 3 \\
\hline $\mathrm{X}$ direction & $\begin{array}{rr}-41.3 & 40.5 \\
30.7 & -111.8\end{array}$ & $\begin{array}{rr}-64.0 & 59.0 \\
43.1 & -158.2\end{array}$ & $\begin{array}{rr}-55.5 & 37.1 \\
16.3 & -170.6\end{array}$ \\
\hline Y direction & $\begin{array}{rr}-73.5 & 44.6 \\
37.9 & -49.1 \\
\end{array}$ & $\begin{array}{r}-114.3 \\
52.8\end{array}$ & $\begin{array}{r}-142.8 \\
85.2\end{array}$ \\
\hline Fixed Posture & $\begin{array}{rr}-53.5 & 40.9 \\
35.7 & -126.5\end{array}$ & & \\
\hline
\end{tabular}

B. Simulated Joint Stiffness

\begin{tabular}{|c|c|c|c|}
\hline & Condition 1 & Condition 2 & Condition 3 \\
\hline $\mathrm{X}$ direction & $\begin{array}{ll}-9.04 & -0.22 \\
-1.33 & -4.73\end{array}$ & $\begin{array}{rr}-14.03 & -0.76 \\
-2.55 & -6.94\end{array}$ & $\begin{array}{rr}-12.29 & -2.36 \\
-4.69 & -9.98\end{array}$ \\
\hline Y direction & $\begin{array}{rr}-16.22 & -3.31 \\
-4.06 & -2.32\end{array}$ & $\begin{array}{rr}-25.26 & -5.37 \\
-7.03 & -4.39\end{array}$ & $\begin{array}{rr}-31.45 & -5.22 \\
-6.54 & -2.72\end{array}$ \\
\hline Fixed Posture & $\begin{array}{rr}-11.76 & -1.59 \\
-2.17 & -5.98\end{array}$ & & \\
\hline
\end{tabular}

Table 2. Simulated stiffness matrices in hand coordinates (A) and in joint coordinates (B). Units are $\mathrm{N} / \mathrm{m}$ and N.m/rad respectively. In Condition 1, shoulder and elbow cocontraction were both $15 \mathrm{~N}$. In Condition 2, the cocontraction command for both shoulder and elbow were set to $30 \mathrm{~N}$. In Condition 3, for movement in $\mathrm{Y}$, elbow cocontraction was $10 \mathrm{~N}$ and shoulder cocontraction was $60 \mathrm{~N}$. For movements in $\mathrm{X}$, elbow and shoulder cocontraction were $50 \mathrm{~N}$ and $20 \mathrm{~N}$ respectively. 


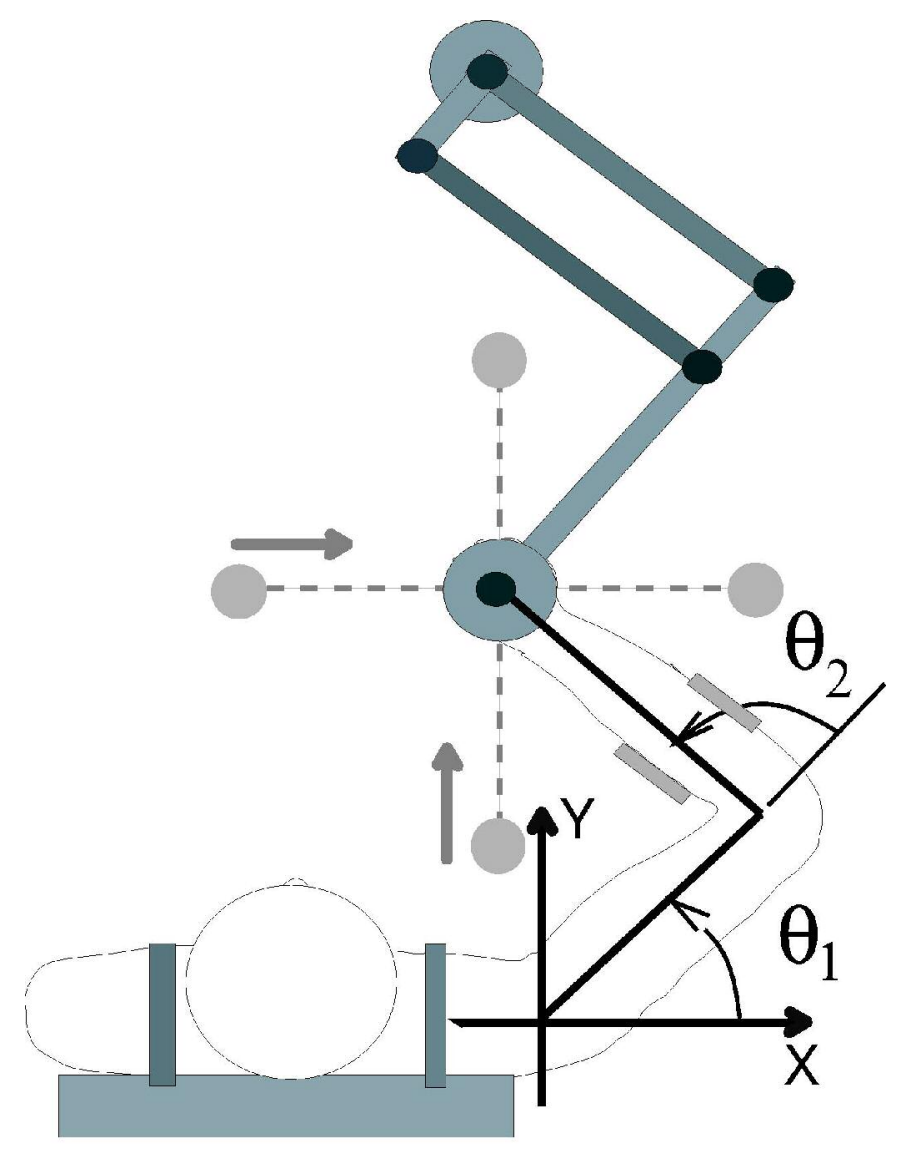

Figure1 

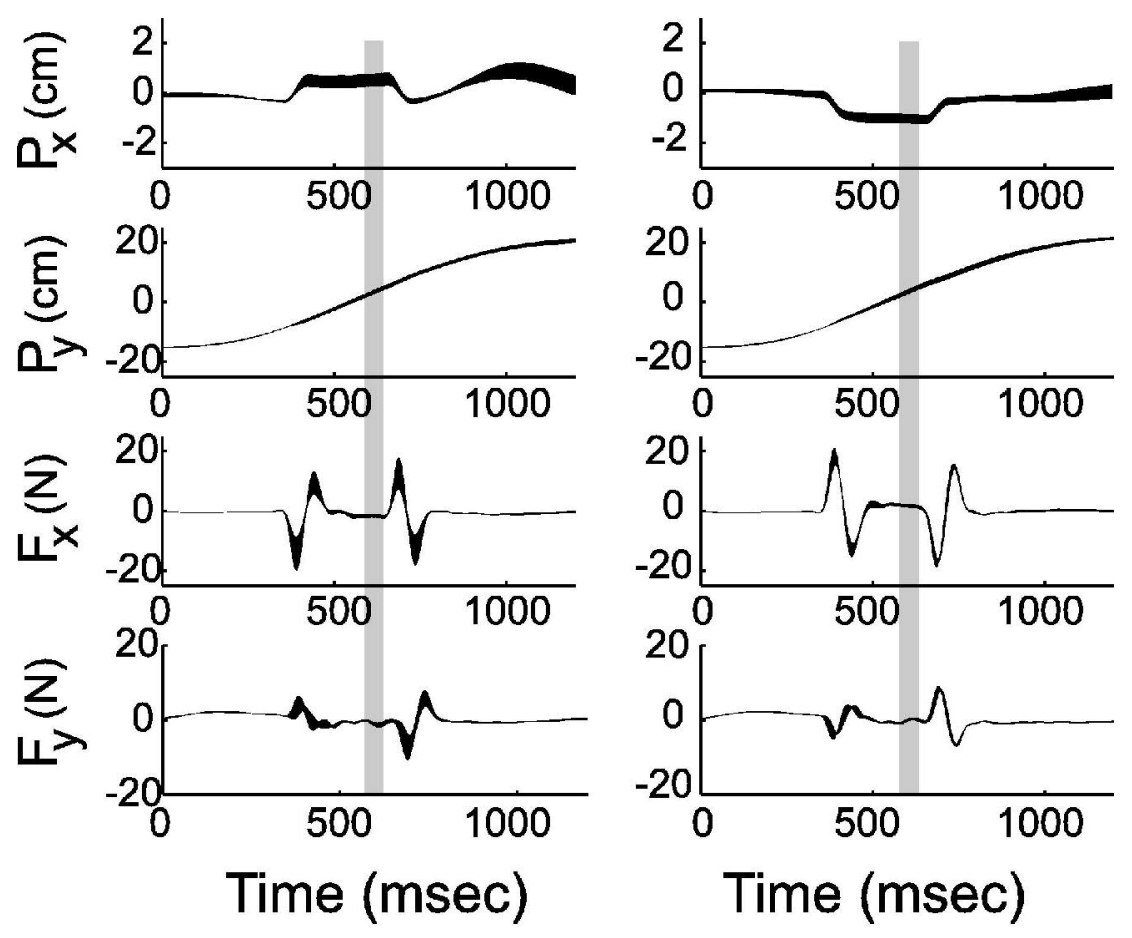

Figure 2 


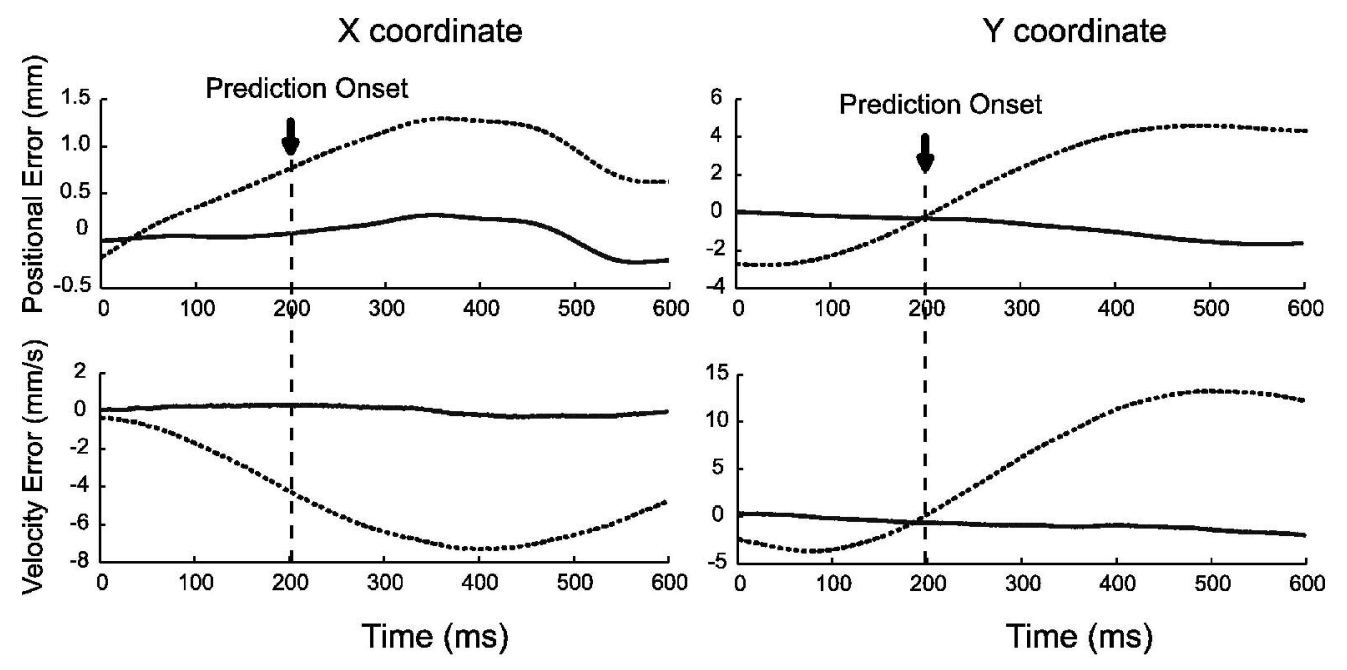

Figure 3 
A
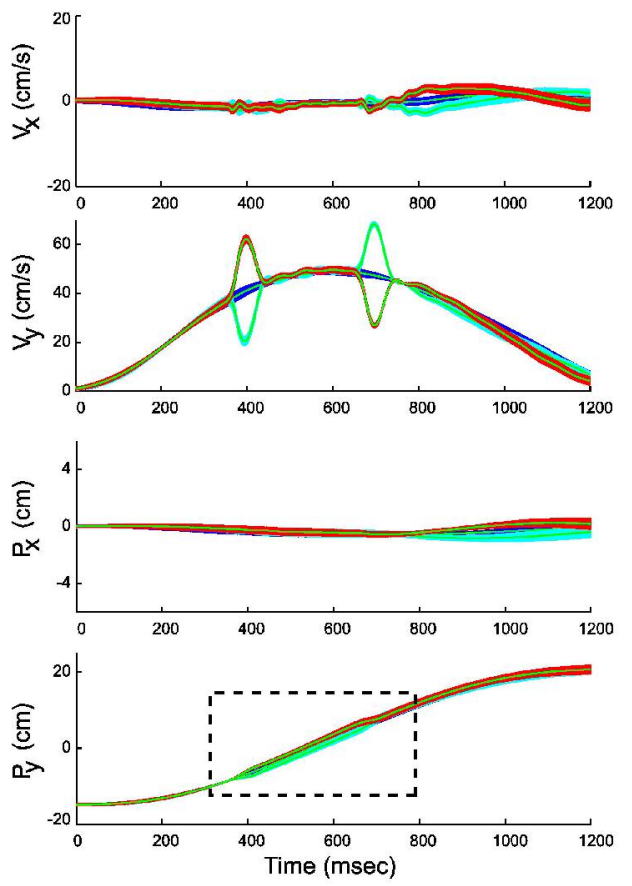

C
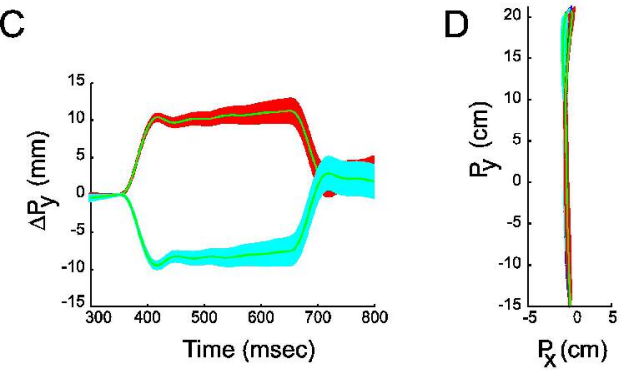

B
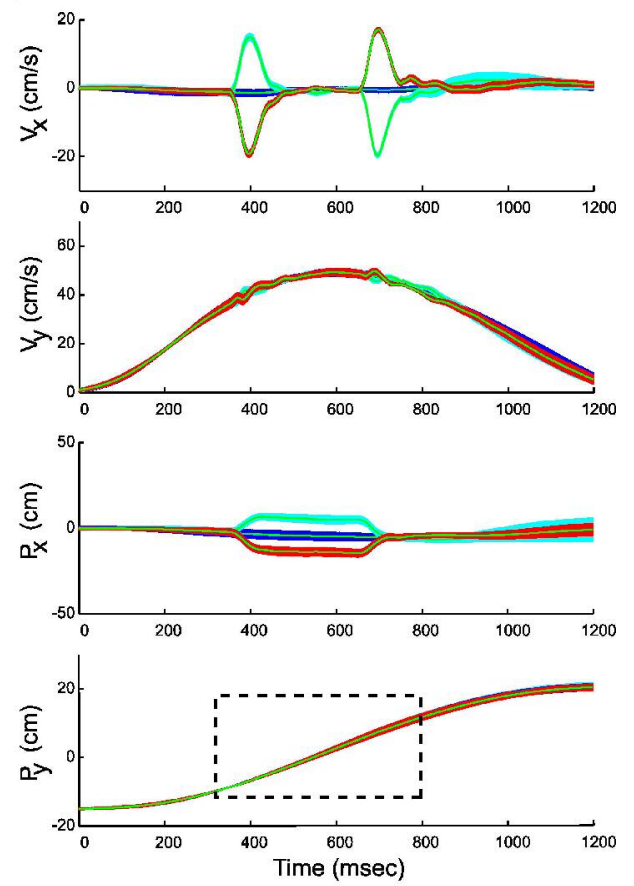

E

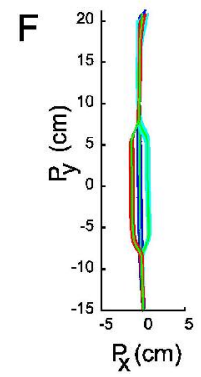

Figure 4 

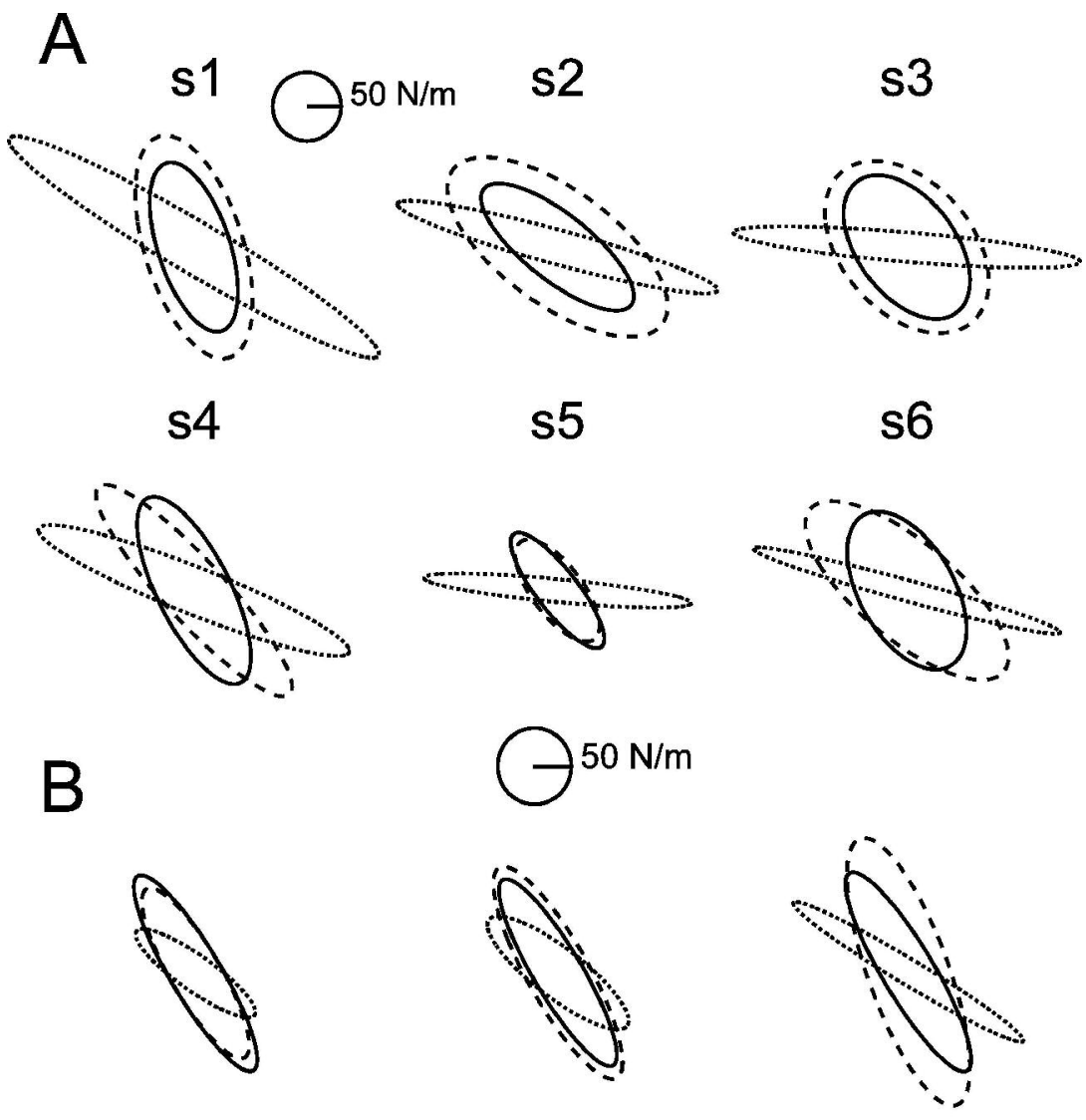

Figure 5 


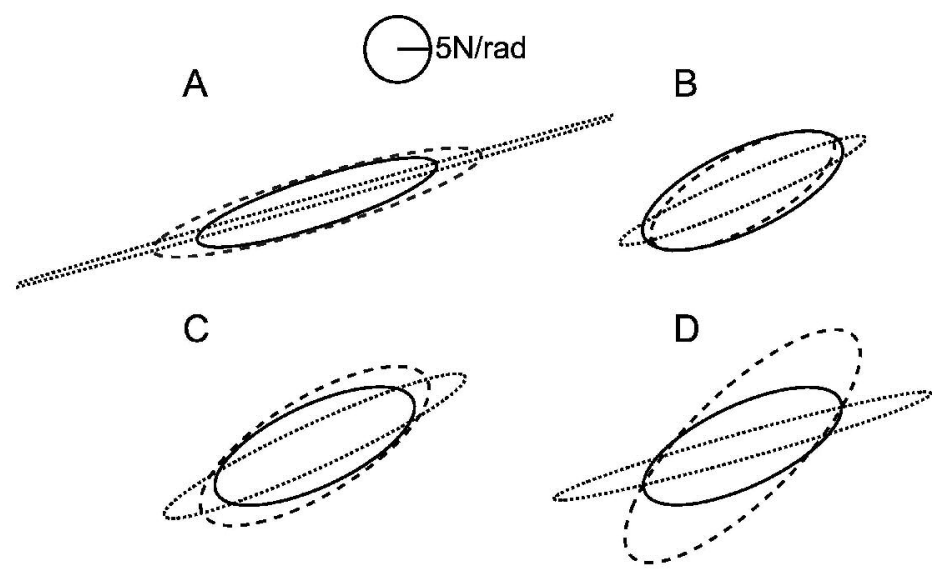

Figure 6 


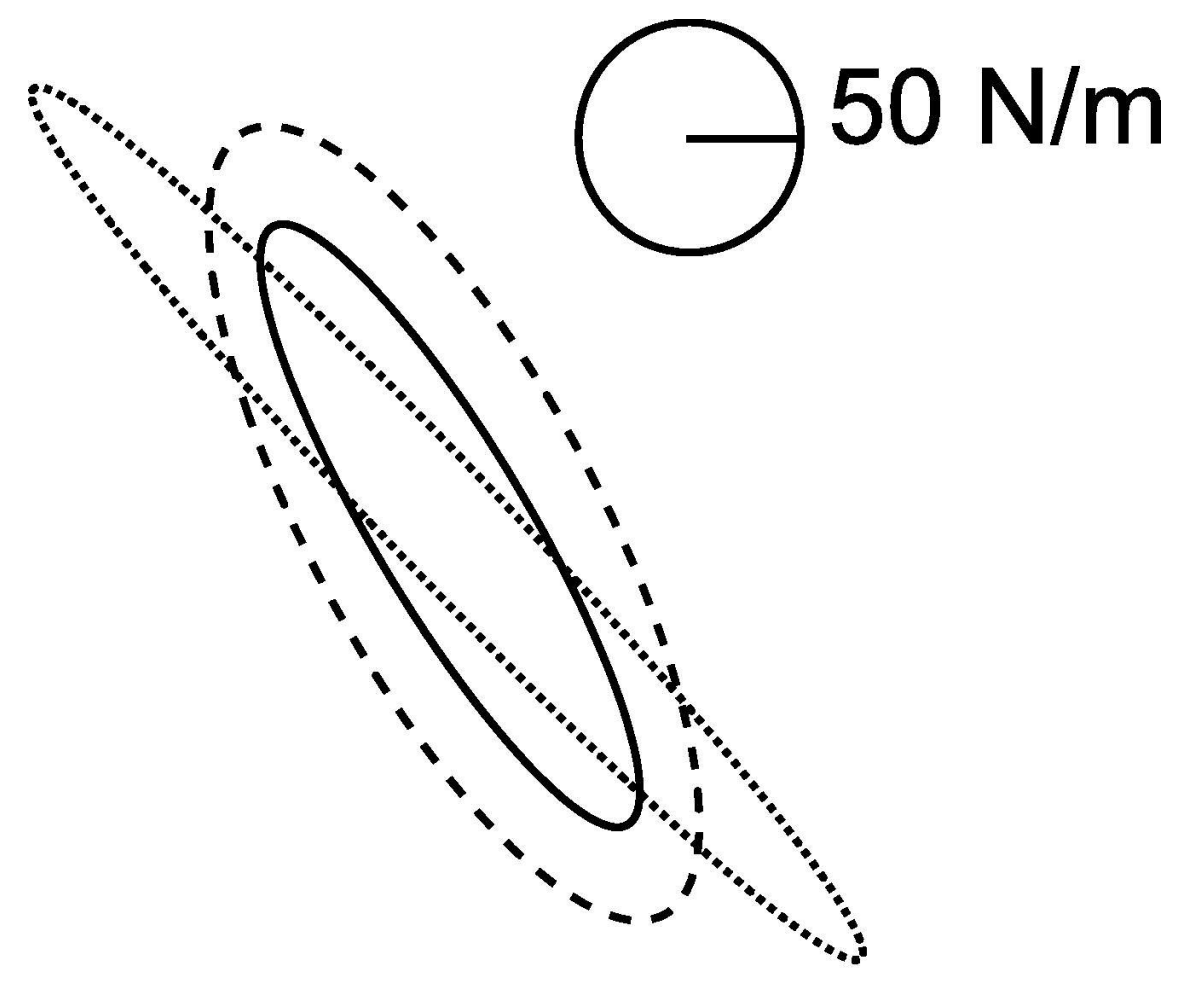

Figure 7 